\title{
Left-sided primary tumors are associated with favorable prognosis in patients with $K R A S$ codon 12/13 wild-type metastatic colorectal cancer treated with cetuximab plus chemotherapy: an analysis of the AIO KRK-0104 trial
}

\author{
J. C. von Einem • V. Heinemann · L. Fischer von Weikersthal $\cdot$ U. Vehling-Kaiser $\cdot$ M. Stauch \\ H. G. Hass · T. Decker $\cdot$ S. Klein $\cdot$ S. Held $\cdot$ A. Jung $\cdot$ T. Kirchner $\cdot$ M. Haas $\cdot$ J. Holch $\cdot$ \\ M. Michl $\cdot$ P. Aubele $\cdot$ S. Boeck $\cdot$ C. Schulz $\cdot$ C. Giessen $\cdot$ S. Stintzing $\cdot$ D. P. Modest
}

Received: 17 March 2014 / Accepted: 28 March 2014 / Published online: 10 May 2014

(C) The Author(s) 2014. This article is published with open access at Springerlink.com

\begin{abstract}
Purpose AIO KRK-0104 investigated first-line therapy of metastatic colorectal cancer (mCRC) with cetuximab, capecitabine and irinotecan versus cetuximab, capecitabine and oxaliplatin. This analysis investigated the impact of primary tumor location on outcome of patients.

Patients and methods Left-sided primary tumors were defined as tumors from rectum to left flexure, while tumors in the remaining colon were regarded right sided. Overall survival (OS), progression-free survival (PFS) and response rate were correlated with primary tumor location. A Cox regression model was used to evaluate interaction between primary tumor location and KRAS mutation.

Results Of 146 patients of the AIO KRK-0104 trial, 100 patients presented left-sided (of those 68 KRAS codon
\end{abstract}

S. Stintzing and D. P. Modest have contributed equally to this article and share last authorship.

J. C. von Einem $(\varangle) \cdot$ V. Heinemann $\cdot$ M. Haas $\cdot$ J. Holch $\cdot$

M. Michl · P. Aubele · S. Boeck · C. Schulz · C. Giessen ·

S. Stintzing $\cdot$ D. P. Modest $(\square)$

Department of Medicine III, University Hospital Grosshadern,

University of Munich, Munich, Germany

e-mail: jobst.von-einem@med.uni-muenchen.de

D. P. Modest

e-mail: dominik.modest@med.uni-muenchen.de

L. F. von Weikersthal

Klinikum St. Marien, Amberg, Germany

U. Vehling-Kaiser

Onkologische Praxis Landshut, Landshut, Germany

M. Stauch

Onkologische Schwerpunktpraxis in Kronach,

Kronach, Germany
12/13 wild-type) and 46 patients right-sided primary tumors (of those 27 KRAS codon 12/13 wild-type). Leftsided tumors were associated with significantly longer OS $(p=0.016, \mathrm{HR}=0.63)$ and PFS $(p=0.02, \mathrm{HR}=0.67)$ as compared to right-sided tumors. These effects were present in the KRAS codon 12/13 wild-type population (HR OS: 0.42; HR PFS: 0.54), while no impact of primary tumor location was evident in patients with KRAS codon 12/13 mutant tumors (HR OS: 1.3; HR PFS: 1.01). A significant interaction of KRAS status and primary tumor location concerning OS and PFS was observed.

Conclusion Our findings suggest that primary tumor location and KRAS codon 12/13 mutational status interact on the outcome of patients with $\mathrm{mCRC}$ receiving cetuximabbased first-line therapy. Left-sided primary tumor location might be a predictor of cetuximab efficacy.

H. G. Hass

Paracelsus Klinik Scheidegg, Scheidegg, Germany

T. Decker

Onkologie Ravensburg, Ravensburg, Germany

S. Klein

Klinikum Bayreuth, Bayreuth, Germany

S. Held

ClinAssess, Gesellschaft für Klinische Forschung mbH, Leverkusen, Germany

A. Jung $\cdot$ T. Kirchner

Department of Pathology, University of Munich, Munich, Germany 
Keywords Colorectal cancer - Primary tumor location . CAPIRI plus cetuximab - CAPOX plus cetuximab . $K R A S$ mutation status

\section{Introduction}

The idea of personalized medicine was introduced to the treatment of metastatic colorectal cancer (mCRC) when KRAS codon 12/13 mutations were identified as negative predictors of anti-EGFR-antibody (EGFR-mAB) treatment. Consequently, only patients with KRAS codon 12/13 wildtype tumors were subjected to cetuximab or panitumumab treatment (Douillard et al. 2013; Huang et al. 2012; Modest et al. 2012; Douillard et al. 2010; Bokemeyer et al. 2011; Amado et al. 2008). This KRAS codon 12/13 wild-type population already excluded about $40 \%$ of all patients and was associated with improved response rates (objective response rates, ORRs), progression-free survival (PFS) and overall survival (OS) in patients receiving EGFR-mABs. However, ORR in clinical trials investigating EGFR-based first-line regimens was usually $<60 \%$, indicating that $K R A S$ codon $12 / 13$ wild-type alone was not a sufficient condition to predict response (Douillard et al. 2013; Modest et al. 2012; Van Cutsem et al. 2011; De Roock et al. 2010; Stintzing et al. 2009). The identification of additional negative predictors such as KRAS exon 3/4 and NRAS exon 2-4 mutations created a new target population for EGFR-mABs: patients with RAS wild-type tumors. This population comprises about $50 \%$ of all patients with $\mathrm{mCRC}$ with a benefit in median OS following EGFR-targeted first-line therapy of 5-7 months (Douillard et al. 2013; Stintzing et al. 2009).

Taking into account that even RAS wild-type tumors potentially do not define the perfect marker for response to EGFR-mABs, additional biomarkers are needed. This question was recently addressed by retrospective evaluations of patients receiving cetuximab treatment in further treatment lines. The efficacy of cetuximab was determined to be modulated by the location of the primary tumor (Missiaglia et al. 2013; Brule et al. 2013). Due to this initial evidence, the question was raised whether the location of the primary tumor in colorectal cancer can serve as a prognostic marker and potentially as a predictive marker for treatment with EGFR-mABs. To our knowledge, the effect of primary tumor location on outcome has not been shown in a mCRC study population receiving first-line treatment with cetuximab.

The AIO KRK-0104 trial randomized patients to CAPIRI plus cetuximab or CAPOX plus cetuximab. With reference to this design, we hypothesized that primary tumor location of the left colon might have a favorable prognostic effect in patients with KRAS wild-type tumors, but not in patients with KRAS mutant tumors.

\section{Methods}

Study design

Data for this analysis were obtained from the AIO KRK0104 trial. This study was a randomized, multicenter phase II trial to investigate the efficacy of cetuximab plus CAPIRI versus cetuximab plus CAPOX as first-line chemotherapy in patients with $\mathrm{mCRC}$ and recruited patients from 2004 to 2006. The primary analysis and the molecular subgroups analysis have been published elsewhere (Modest et al. 2012; Moosmann et al. 2011). Primary endpoint of the AIO KRK-0104 study was ORR. This investigation refers to the population of 146 patients with central assessment of $K R A S / B R A F$ mutations as published before (Modest et al. 2012).

\section{Definition of right-sided versus left-sided tumors}

The primary tumor location was defined in the study reports and was extracted from the central database. Tumors located in rectum, sigma, descending colon and the left flexure were defined as left-sided tumors. All tumors from cecum to the distal part of the transverse colon were categorized as right-sided tumors.

\section{Treatment schedule}

In both arms, cetuximab was given at an initial dose of $400 \mathrm{mg} / \mathrm{m}^{2}$ as a 120 -min infusion, followed by weekly infusions of $250 \mathrm{mg} / \mathrm{m}^{2}$ over $60 \mathrm{~min}$. Patients in arm A received chemotherapy with CAPIRI (i.e., oral capecitabine $800 \mathrm{mg}$ / $\mathrm{m}^{2}$ twice daily on days 1 through 14 , followed by a 1 -week rest period plus irinotecan $200 \mathrm{mg} / \mathrm{m}^{2}$ as a 30 -min intravenous infusion on day 1). In patients older than 65 years, doses were further reduced by $20 \%$. Patients in arm B received chemotherapy with CAPOX (i.e., capecitabine $1,000 \mathrm{mg} / \mathrm{m}^{2}$ twice daily on days 1 through 14 , followed by a 1 -week rest period plus oxaliplatin $130 \mathrm{mg} / \mathrm{m}^{2}$ as a 120 min intravenous infusion on day 1). Treatment cycles were repeated every 3 weeks until disease progression or unacceptable toxicity (Moosmann et al. 2011).

\section{Patients}

The patient population of the AIO KRK-0104 trial was described in recent reports (Modest et al. 2012; Moosmann et al. 2011). Patients with $B R A F$ mutant tumors were analyzed as $K R A S$ wild-type tumors. One patient presenting a tumor with $B R A F$ and $K R A S$ mutation was regarded as KRAS mutant in this analysis. In two patients, two primary tumors were located in the left-sided colon (sigma and rectum; descendent colon and sigma); these cases were 
analyzed as left-sided colorectal cancer. In another patient, one primary tumor was located in the right part of the colon (cecum), while another primary tumor was observed at the left side (rectum); this case was classified as right-sided colorectal cancer.

\section{Endpoints}

The present investigation was performed as an exploratory analysis using response rates $(\mathrm{ORR}=$ complete and partial remission), PFS and OS as parameters for outcome in patients with tumors of right-sided and left-sided origin. Tumor assessment was performed every two cycles (6 weeks). A final update on overall survival was conducted in 2011, and the statistical analysis plan was published in detail (Modest et al. 2012; Moosmann et al. 2011).

\section{Statistical analysis}

In this retrospective, exploratory investigation, OS and PFS were stratified by primary tumor location and were estimated using the Kaplan-Meier method. Possible differences were evaluated by log-rank test and Cox regression analysis. A Cox regression model was used to evaluate interaction between primary tumor location and KRAS mutation as explanatory variables. $\chi^{2}$ tests compared response rates. A $p$ value $<0.05$ was regarded significant. For interaction test, a $p$ value $<0.10$ was regarded significant. SPSS PASW 21.0 (SPSS Inc., Chicago, Illinois) and SAS 9.2 (SAS Institute Inc., Cary, NC, USA) were used for statistical analysis.

\section{KRAS mutation detection}

$K R A S / B R A F$ testing was performed in a German reference laboratory for KRAS analysis (University of Munich, Department of Pathology) as described before (Modest et al. 2012; Moosmann et al. 2011).

\section{Results}

Study population and tumor characteristics

In all 146 patients of the pathological analysis-set, the primary tumor location was assessable. Out of the full population, 100 patients presented with left-sided tumors, whereas 46 patients presented primary right-sided tumors. In detail, tumors were located in rectum $(n=49)$, sigma $(n=40)$, descending colon $(n=7)$, left flexure $(n=2)$, transverse colon $(n=11)$, ascending colon $(n=18)$, cecum $(n=16)$ and double primary location $(n=3)$. Out of the 100 patients presenting left-sided colorectal tumors,
68 tumors presented KRAS codon 12/13 wild-type tumors, and 32 tumors had KRAS codon 12/13 mutations. Out of 46 tumors of right-sided origin, 27 tumors were diagnosed with $K R A S$ codon $12 / 13$ wild-type status, while 19 patients presented a KRAS codon 12/13 mutant tumor. Distribution of patients with left-sided versus right-sided tumors to the treatment arms of the AIO KRK-0104 trial (CAPIRI plus cetuximab/CAPOX plus cetuximab) was comparable (52/48 \% vs. 50/50 \%; Table 1$)$.

\section{Baseline patient characteristics}

Baseline patient characteristics are shown in Table 1. No major imbalances associated with primary tumor location in the left or right part of the colon were present in our cohort. However, a trend toward more female patients was observed in the group of right-sided tumors when compared to the group of patients with left-sided primary tumors (39\% vs. $23 \%, p=0.05$; Table 1 ).

Effect of primary tumor location on overall survival (OS)

The whole study population reached a median OS of 21.1 months. Survival times by exact tumor locations are shown in Fig. 1a. If analyzed as right vs. left colon, median OS of patients with right-sided tumor was 14.8 months, while median OS in patients with left-sided tumor was 26.3 months $(p=0.016, \mathrm{HR}=0.63)$, (Fig. 1b). In patients with $K R A S$ codon $12 / 13$ wild-type tumors, median OS was 13.0 months in patients with right-sided versus 29.0 months in patients with left-sided mCRC $(p<0.001, \mathrm{HR}: 0.42)$. The effect of primary tumor location on OS in patients with $B R A F$ V600E mutant tumors seemed consistent with the observation in the KRAS codon 12/13 wild-type cohort. In patients with KRAS codon 12/13 mutant tumors, no significant difference was present when OS of patients with rightsided and left-sided mCRC was compared (Figs. 1c-d and 3a).

Effect of primary tumor location on progression-free survival (PFS)

Median PFS in all patients was 7.0 months. PFS by exact tumor location is shown in Fig. 2a. In patients with rightsided tumors, median PFS was 5.2 months, and in patients with left-sided-tumors, it was 7.8 months $(p=0.02$, $\mathrm{HR}=0.67$ ). If $K R A S$ status was taken into account, in accordance to OS, a significant difference in PFS associated with primary tumor location was only evident in patients with KRAS codon 12/13 wild-type tumors (4.6 vs. 8.4 months, $p=0.007$, HR $=0.54$ ), but not in patients presenting a mutation in these loci. In patients with $B R A F$ mutant mCRC, the effect of primary tumor location seemed 
Table 1 Baseline characteristics of patients and tumors

\begin{tabular}{|c|c|c|c|c|c|}
\hline & \multicolumn{2}{|c|}{$\begin{array}{l}\text { Patients with } \\
\text { left-sided } \\
\text { mCRC }\end{array}$} & \multicolumn{2}{|c|}{$\begin{array}{l}\text { Patients with } \\
\text { right-sided } \\
\text { mCRC }\end{array}$} & \multirow[t]{2}{*}{$p$ value } \\
\hline & $n$ & $\%$ & $n$ & $\%$ & \\
\hline Patients & 100 & 68 & 46 & 32 & \\
\hline \multicolumn{6}{|l|}{ Age } \\
\hline Median & \multicolumn{2}{|c|}{63} & \multicolumn{2}{|c|}{61} & 0.50 \\
\hline Range & \multicolumn{2}{|c|}{$32-77$} & \multicolumn{2}{|c|}{$47-74$} & \\
\hline \multicolumn{6}{|l|}{ Sex } \\
\hline Female & 23 & 23 & 18 & 39 & 0.05 \\
\hline Male & 77 & 77 & 28 & 61 & \\
\hline \multicolumn{6}{|l|}{ Performance status (Karnofsky) } \\
\hline $100+90$ & 73 & 73 & 32 & 70 & 0.55 \\
\hline $80+70$ & 25 & 25 & 14 & 30 & \\
\hline Not reported & 2 & 2 & 0 & 0 & \\
\hline \multicolumn{6}{|l|}{ Prior therapy } \\
\hline Chemotherapy & 21 & 21 & 5 & 11 & 0.17 \\
\hline Radiotherapy & 12 & 12 & 1 & 2 & 0.06 \\
\hline \multicolumn{6}{|l|}{ Disease sites } \\
\hline Liver & 84 & 84 & 42 & 91 & 0.31 \\
\hline Lung & 32 & 32 & 19 & 41 & 0.35 \\
\hline Lymph node & 30 & 30 & 20 & 43 & 0.13 \\
\hline Peritoneum & 12 & 12 & 3 & 7 & 0.39 \\
\hline \multicolumn{6}{|l|}{ Treatment arm } \\
\hline CAPIRI plus cetuximab & 52 & 52 & 23 & 50 & 0.86 \\
\hline CAPOX plus cetuximab & 48 & 48 & 23 & 50 & \\
\hline \multicolumn{6}{|l|}{ Tumor mutation status } \\
\hline$K R A S$ codon $12 / 13$ wild-type & 68 & 68 & 27 & 59 & \multirow{2}{*}{0.35} \\
\hline$K R A S$ codon $12 / 13$ mutant & 32 & 32 & 19 & 41 & \\
\hline$B R A F$ V600E mutant & 6 & 6 & 11 & 24 & 0.004 \\
\hline
\end{tabular}

$p$ values: Chi-square test/Fisher's exact test except for age: MannWhitney $U$ test

again consistent with the effects observed in patients with $K R A S$ codon 12/13 wild-type tumors (Figs. $2 \mathrm{~b}-\mathrm{d}$ and $3 \mathrm{~b}$ ).

Interaction of KRAS codon 12/13 mutation and primary tumor location

Cox regression models with hazard ratios for primary tumor location, KRAS mutation and interaction respectively as explanatory variables were analyzed. For OS, the hazard ratio of primary tumor location was 0.160 in favor of left-sided ( $p=0.002,95 \%$ CI $0.050-0.511), 0.640$ in favor of wild-type ( $p=0.159,95 \%$ CI 0.344-1.191) for KRAS mutation, and $0.372(p=0.013,95 \%$ CI $0.171-0.810)$ for interaction between primary tumor location and KRAS mutation. For PFS, the hazard ratio of primary tumor location was 0.252 in favor of left-sided tumors $(p=0.013$, $95 \%$ CI $0.085-0.745), 0.780$ in favor of wild-type $(p=0.411,95 \%$ CI $0.432-1.140)$ for KRAS mutation, and 0.487 ( $p=0.056,95 \%$ CI $0.233-1.017$ ) for interaction between primary tumor location and KRAS mutation.

Influence of $B R A F \mathrm{~V} 600 \mathrm{E}$ mutations in this study

In our cohort, BRAF mutation was more frequent in rightsided compared with left-sided primary tumors (24 vs. $6 \%$ ). After removing $B R A F$ V600E mutant tumors from the KRAS codon 12/13 wild-type cohort, median PFS of patients with $K R A S / B R A F$ wild-type tumors $(n=79)$ was 5.9 months in patients with right-sided versus 8.2 months with left-sided tumors $(p=0.47, \mathrm{HR}=0.81)$. Median OS was 16.2 months in patients with right-sided compared with 27.3 months in patients with left-sided tumors $(p=0.11$, $\mathrm{HR}=0.60$; Fig. 3a, b).

Effect of primary tumor location on response rate

Response rates were analyzed based on non-missing data and did not show significant differences. ORR was $58 \%$ in left-sided and $53 \%$ in right-sided tumors $(p=0.70)$ in the whole study population. In KRAS codon 12/13 wild-type tumors, right-sided tumors were associated with a lower ORR when compared to left-sided tumors ( $43 \%$ vs. $64 \%$ ), and this difference did not reach significance $(p=0.12)$. When comparing response rates in patients with KRAS codon $12 / 13$ mutant mCRC, ORR was $65 \%$ in right-sided and $45 \%$ in left-sided tumors $(p=0.23$; Table 2$)$.

\section{Discussion}

Personalized treatment of mCRC patients is entering daily routine in clinical practice. The more tumor sub-classifications based on molecular markers are defined, the higher the chance is to identify positive and negative predictors and consequently to specify different strategies of therapy. Clinical data have proven that mutant RAS genes are negative predictive biomarkers and that patients with a KRAS/NRAS mutation do not benefit from an EGFR-mABbased therapy (De Roock et al. 2010; Peeters et al. 2013a, b; Andre et al. 2013). Therefore, mutations of KRAS and $N R A S$ represent an established negative predictor of EGFR$\mathrm{mAB}$ efficacy. The role of $B R A F$ in first-line treatment of mCRC is described as a negative prognostic marker, but not as a predictive marker in terms of EGFR-mAB therapy (Douillard et al. 2013). Recently, it has been suggested that, in addition to RAS mutations, the primary tumor location might play a crucial role for efficacy of EGFR-mABs (Douillard et al. 2013; Missiaglia et al. 2013).

For this reason, we hypothesized that in the AIO KRK0104 trial primary tumor location in the left colon might 

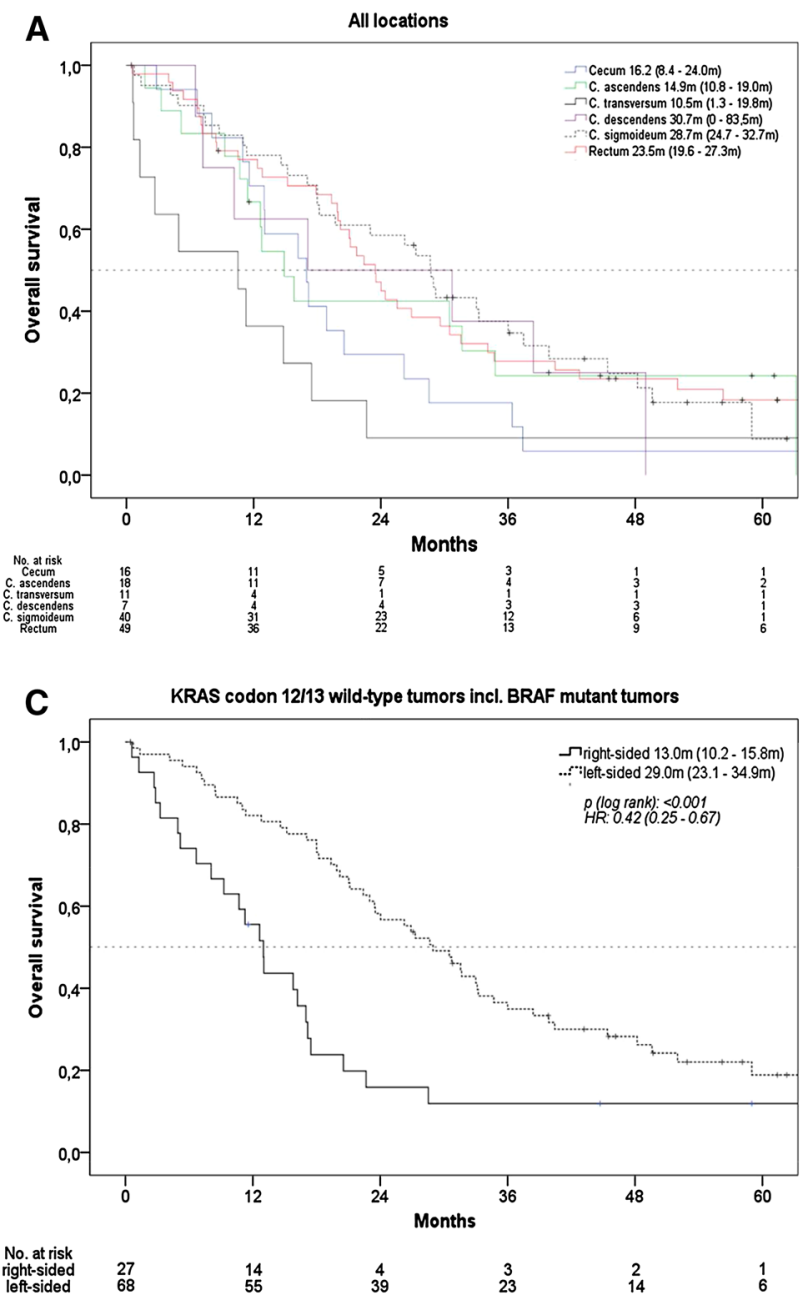

Fig. 1 Overall survival (OS), a according to exact primary tumor location, patients with double primary tumors and with primary tumor of left flexure were excluded due to sample size, $\mathbf{b}$ according

have a favorable prognostic effect in patients with KRAS wild-type tumors, but not in patients with KRAS mutant tumors when receiving cetuximab-based first-line therapy. In fact, OS and PFS differed significantly when comparing left- to right-sided tumors. This effect was driven by patients with KRAS codon $12 / 13$ wild-type tumors and seemed also present in those patients that presented with KRAS codon 12/13 wild-type but BRAF V600E mutant tumors. By contrast, in patients with KRAS codon 12/13 mutant tumors, the primary tumor location was not associated with significant differences in terms of OS or PFS. This interaction of KRAS mutation and primary tumor location was found to be significant for both OS and PFS. No significant impact of primary tumor location on response rates was observed in this study. Taking the difference in patients with KRAS wild-type tumors into account (64\% vs. $43 \%$ in patients with left-sided vs right-sided primary
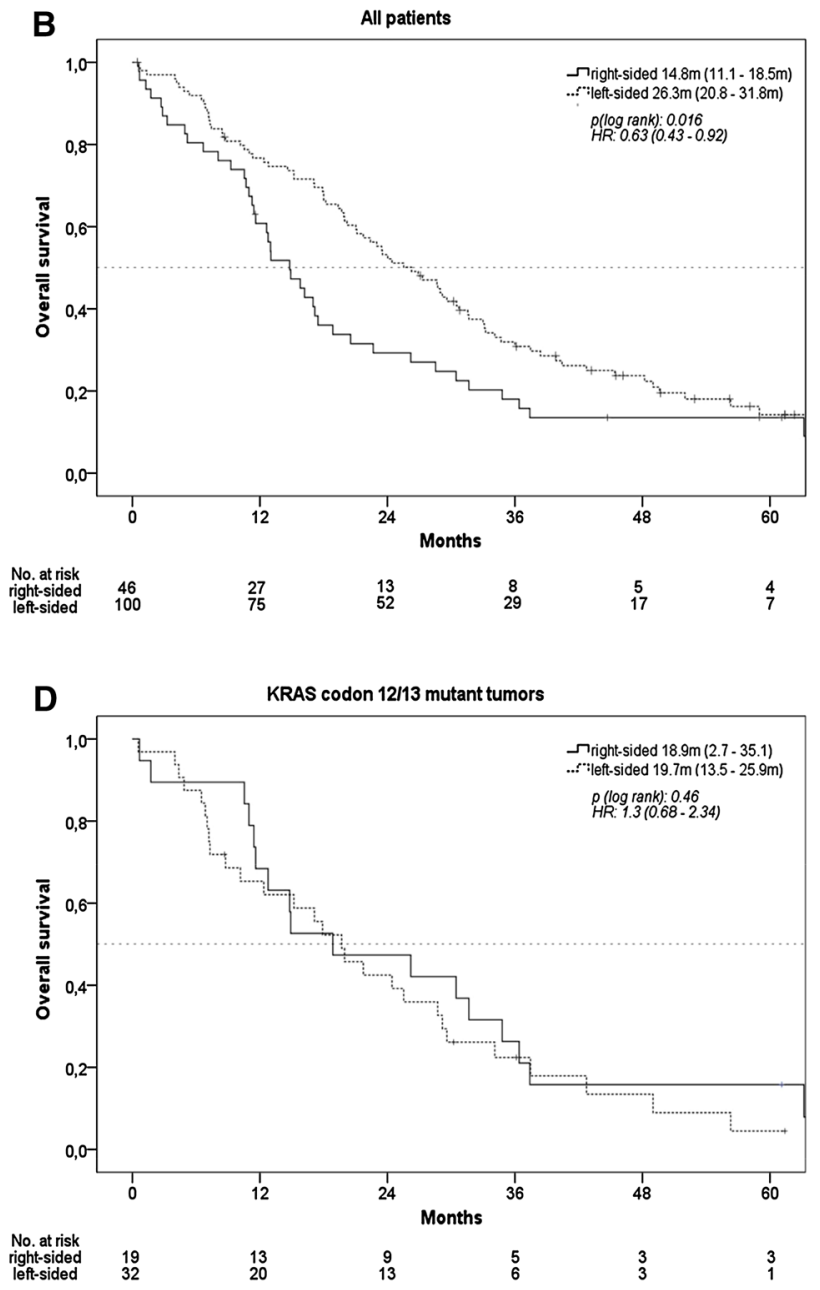

to right-sided versus left-sided mCRC, $\mathbf{c}$ patients with KRAS codon $12 / 13$ wild-type tumors, d patients with KRAS codon 12/13 mutant tumors

tumor), this could be interpreted as a consequence of missing sample size for this endpoint.

Our results are supported by a recent analysis of the NCIC CTG CO.17 trial (that investigated cetuximab plus best supportive care (BSC) versus BSC alone), which reported less striking cetuximab-induced effects in patients with KRAS codon 12/13 wild-type, right-sided tumors as compared with patients bearing a left-sided tumor (Brule et al. 2013). A similar observation was reported by Missiaglia and colleagues who observed a longer PFS in refractory patients that received cetuximab treatment if the primary tumor was left-sided as compared to right-sided tumors (Missiaglia et al. 2013).

As described above, we grouped patients in left-sided versus right-sided tumors, which included tumors from cecum to the distal part of the transverse colon. This distinction corresponds to the midgut versus hindgut definition 

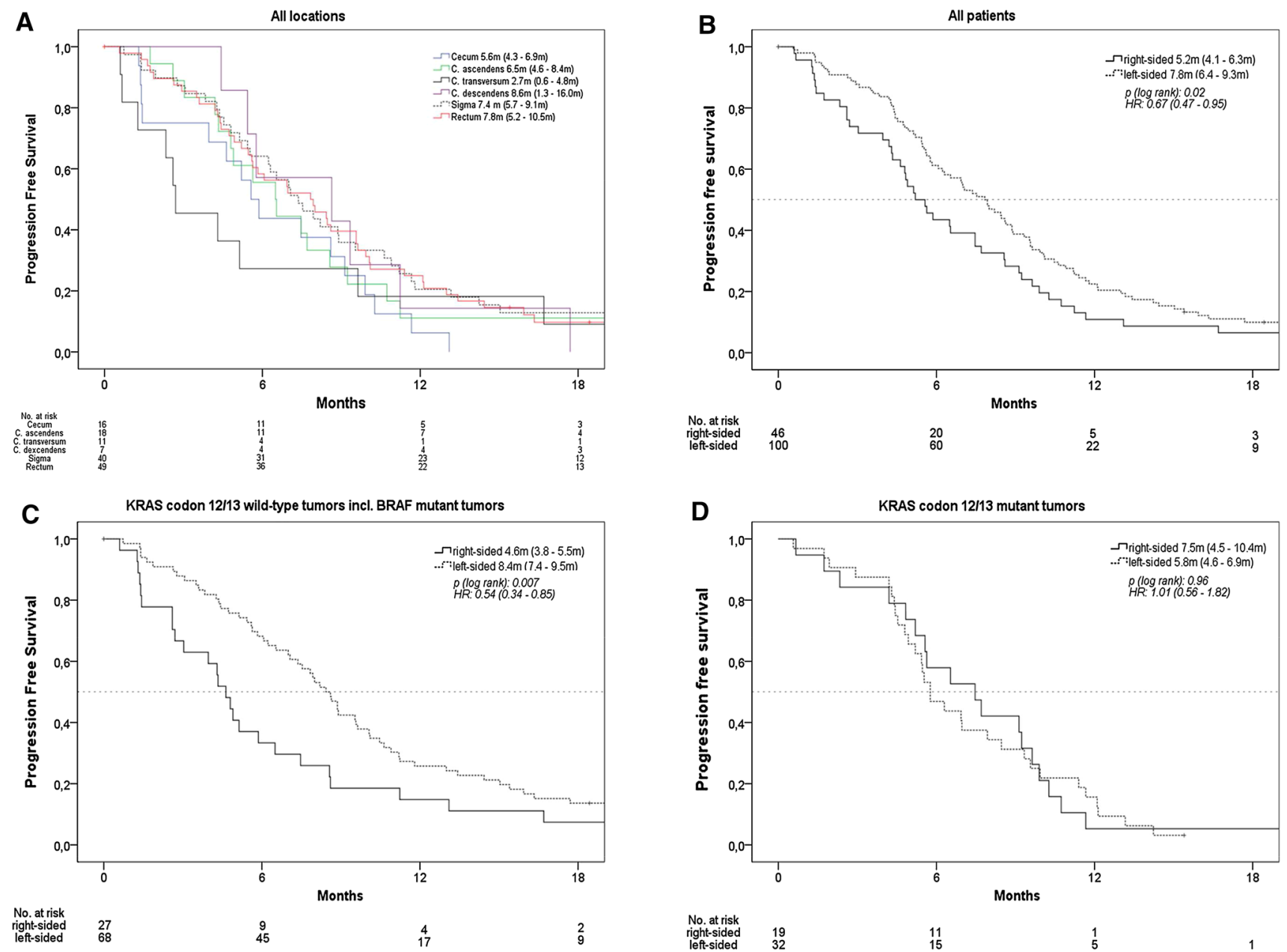

Fig. 2 Progression-free survival (PFS), a according to exact primary tumor location patients with double primary tumors and with primary tumor of left flexure were excluded due to sample size, $\mathbf{b}$ according

to right-sided versus left-sided mCRC, $\mathbf{c}$ patients with KRAS codon $12 / 13$ wild-type tumors, d patients with KRAS codon 12/13 mutant tumors

Fig. 3 Hazard ratios of molecular subgroups, error bars indicating the $95 \%$ confidence interval. a Overall survival, b progression-free survival a

Overall survival

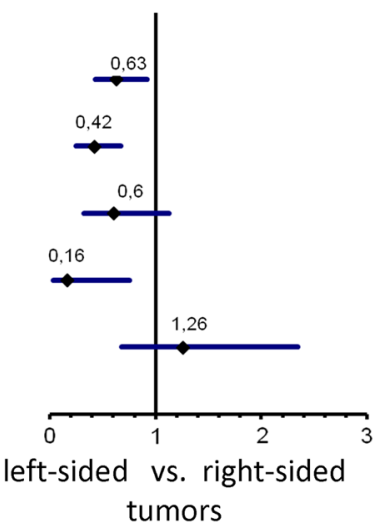

b Progression-free survival

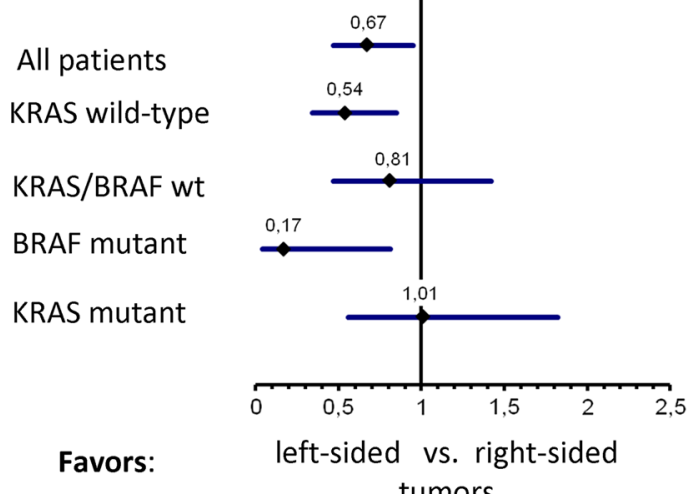

and is modified counting the total colon transversum as right-sided colon. Nevertheless, the strict separation of different tumor locations is questioned by the "continuum hypothesis," which postulates that molecular features of the tumor gradually change along bowel subsides, rather than change abruptly at splenic flexure (Yamauchi et al. 2012a, 
Table 2 Response to treatment

\begin{tabular}{lllllll}
\hline Parameter & L-mCRC & R-mCRC & L-mCRC KRAS wt & R-mCRC KRAS wt & L-mCRC KRAS mut & R-mCRC KRAS mut \\
\hline No. of patients & 100 & 46 & 68 & 27 & 32 & 19 \\
ORR evaluable (no. of pts) & 85 & 38 & 56 & 21 & 29 & 17 \\
n.a. (no. of pts) & 15 & 8 & 12 & 6 & 3 & 2 \\
ORR \% (95 \% CI) & $58(47-68)$ & $53(37-68)$ & $64(51-77)$ & $43(24-65)$ & $45(27-65)$ & $65(40-86)$ \\
$p$ value & 0.70 & & 0.12 & & 0.23 & \\
\hline
\end{tabular}

$R$ - $m C R C$ patients with right-sided $\mathrm{mCRC}, L-m C R C$ patients with left-sided mCRC, $w t$ wild-type, $m u t$ mutant; ORR (CR $+\mathrm{PR})$ overall response rate, $C I$ confidence interval, n.a not assessable due to any reason. $p$ value: Fisher's exact test. ORR calculation based on non-missing data (patients evaluable for response)

b). As shown in Figs. 1a and 2a, a trend toward specific OS and PFS could possibly be derived from the exact primary tumor location of the colon, but possibly not according to the physiological course of the colon. Clearly, our data concerning this issue are limited by sample size.

As samples from the AIO KRK-0104 trial were only tested for KRAS exon 2 codon 12/13 mutations, but not for KRAS exon 3, 4 or NRAS mutations, our data might contain a bias of approximately $10 \%$ hidden mutations that we cannot identify due to lacking tumor material. In our cohort, the impact of left- versus right-sided tumors was specifically strong in BRAF mutant tumors. This finding might be explainable by sample size and MSI/MSS status that is unknown for the tumors of AIO KRK-0104 trial cohort. It might have been suspected that the whole side effect might be influenced by $B R A F / M S I / M S S$ status, since $B R A F$ mutations are known to be more frequent in rightsided colorectal cancer (Pai et al. 2012; Popovici et al. 2013). However, even after excluding $B R A F$ mutant tumors from the KRAS codon 12/13 wild-type cohort, the strong prognostic effect of left-sided primary tumors seemed still present (hazard ratio for OS: 0.60). It might be concluded that in mCRC BRAF mutation interacts in the left- versus right-sided tumor story, but is not the only reason for the observed differences.

Our data are limited by several aspects. As discussed above, we distinguished between KRAS mutant and nonmutant only and did not take other RAS mutations into account. Furthermore, this study only consists of a rather small population that might lead to biases, especially in the KRAS mutant cohort. Furthermore, treatment differences between oxaliplatin- and irinotecan-treated patients could not be excluded.

In conclusion, our data correspond favorably with other publications investigating EGFR-mAB use and primary tumor location in mCRC. The interaction of primary tumor location and KRAS mutations suggests that primary tumor location might be an additional biomarker for EGFRmABs. Corresponding pathological findings to explain this phenomenon are still lacking and could be more complex than RAS mutations (Missiaglia et al. 2013; Maus et al. 2013). Data from randomized phase III trials such as CRYSTAL, PRIME and FIRE 3 are necessary to draw definite conclusions concerning the restriction of EGFR-mAbs to patients with RAS wild-type left-sided mCRC.

Acknowledgments We thank all of the patients and their families, the participating centers of this trial and the entire medical staff who contributed to patient care and data collection. We also thank Matthias Wolff for expert secretarial help and organization. S. Stintzing is currently granted a postdoctoral fellowship by the German Cancer Aid (Mildred Scheel Foundation). The AIO KRK-0104 trial was supported by Merck Serono, Roche and Pfizer.

Conflict of interest D.P.M.: research grant, travel support and honoraria for lectures: Merck Serono, Roche, Amgen. Advisory boards: Amgen; V.H.: research grant, travel support, advisory boards and honoraria for lectures: Merck Serono, Roche, Amgen; S.S: research grant, travel support and honoraria for lectures: Merck Serono, Roche, Amgen. Advisory boards: Merck Serono, BMS; CG: travel support: Roche; M.M.: travel support: SIRTeX; P.A.: travel support: Pharma Mar; M.H.: travel support, honoraria for lectures: Celgene, Roche; T.D.: travel support and honoraria for lectures: Roche, Amgen and All other authors have declared no conflict of interest.

Open Access This article is distributed under the terms of the Creative Commons Attribution License which permits any use, distribution, and reproduction in any medium, provided the original author(s) and the source are credited.

\section{References}

Amado RG, Wolf M, Peeters M, Van Cutsem E, Siena S, Freeman DJ et al (2008) Wild-type KRAS is required for panitumumab efficacy in patients with metastatic colorectal cancer. J Clin Oncol 26(10): 1626-1634

Andre T, Blons H, Mabro M, Chibaudel B, Bachet JB, Tournigand C et al (2013) Panitumumab combined with irinotecan for patients with KRAS wild-type metastatic colorectal cancer refractory to standard chemotherapy: a GERCOR efficacy, tolerance, and translational molecular study. Ann Oncol 24(2):412-419

Bokemeyer C, Bondarenko I, Hartmann JT, de Braud F, Schuch G, Zubel A et al (2011) Efficacy according to biomarker status of cetuximab plus FOLFOX-4 as first-line treatment for metastatic colorectal cancer: the OPUS study. Ann Oncol 22(7): $1535-1546$ 
Brule SY, Jonker DJ, Karapetis CS, O'Callaghan CJ, Moore MJ, Wong R, et al. (2013) Location of colon cancer (right-sided [RC] versus left-sided [LC]) as a predictor of benefit from cetuximab (CET): NCIC CTG CO.17. J Clin Oncol 31, (suppl; abstr 3528). Epub 2013 ASCO Annual Meeting

De Roock W, Jonker DJ, Di Nicolantonio F, Sartore-Bianchi A, Tu D, Siena $S$ et al (2010) Association of KRAS p. G13D mutation with outcome in patients with chemotherapy-refractory metastatic colorectal cancer treated with cetuximab. JAMA 304(16):1812-1820

Douillard JY, Siena S, Cassidy J, Tabernero J, Burkes R, Barugel M et al (2010) Randomized, phase III trial of panitumumab with infusional fluorouracil, leucovorin, and oxaliplatin (FOLFOX4) versus FOLFOX4 alone as first-line treatment in patients with previously untreated metastatic colorectal cancer: the PRIME study. J Clin Oncol 28(31):4697-4705

Douillard JY, Oliner KS, Siena S, Tabernero J, Burkes R, Barugel M et al (2013) Panitumumab-FOLFOX4 treatment and RAS mutations in colorectal cancer. N Engl J Med 369(11):1023-1034

Huang F, Xu LA, Khambata-Ford S (2012) Correlation between gene expression of IGF-1R pathway markers and cetuximab benefit in metastatic colorectal cancer. Clin Cancer Res 18(4):1156-1166

Maus M, Hanna D, Stephens C, Grimminger P, Epstein M, Astrow S, et al. (2013) Gene expression profiles and tumor locations in colorectal cancer (left vs. right vs. rectum). J Clin Oncol 31, (suppl; abstr 3527)

Missiaglia E, Jacobs B, Di Narzo AF, Soneson C, Roth A, Bosman F, et al. (2013) Proximal and distal colon tumors as distinct biologic entities with different prognoses. J Clin Oncol 31, 2013 (suppl; abstr 3526). Epub 2013 ASCO Annual Meeting

Modest DP, Jung A, Moosmann N, Laubender RP, Giessen C, Schulz $C$ et al (2012) The influence of KRAS and BRAF mutations on the efficacy of cetuximab-based first-line therapy of metastatic colorectal cancer: an analysis of the AIO KRK-0104-trial. Int J Cancer 131(4):980-986

Moosmann N, von Weikersthal LF, Vehling-Kaiser U, Stauch M, Hass HG, Dietzfelbinger H et al (2011) Cetuximab plus capecitabine and irinotecan compared with cetuximab plus capecitabine and oxaliplatin as first-line treatment for patients with metastatic colorectal cancer: AIO KRK-0104-a randomized trial of the German AIO CRC study group. J Clin Oncol 29(8):1050-1058

Pai RK, Jayachandran P, Koong AC, Chang DT, Kwok S, Ma L et al (2012) BRAF-mutated, microsatellite-stable adenocarcinoma of the proximal colon: an aggressive adenocarcinoma with poor survival, mucinous differentiation, and adverse morphologic features. Am J Surg Pathol 36(5):744-752

Peeters M, Oliner KS, Parker A, Siena S, Van Cutsem E, Huang J et al (2013a) Massively parallel tumor multigene sequencing to evaluate response to panitumumab in a randomized phase III study of metastatic colorectal cancer. Clin Cancer Res 19(7):1902-1912

Peeters M, Douillard JY, Van Cutsem E, Siena S, Zhang K, Williams $\mathrm{R}$ et al (2013b) Mutant KRAS codon 12 and 13 alleles in patients with metastatic colorectal cancer: assessment as prognostic and predictive biomarkers of response to panitumumab. J Clin Oncol 31(6):759-765

Popovici V, Budinska E, Bosman FT, Tejpar S, Roth AD, Delorenzi M (2013) Context-dependent interpretation of the prognostic value of BRAF and KRAS mutations in colorectal cancer. BMC Cancer 13:439

Stintzing S, Heinemann V, Moosmann N, Hiddemann W, Jung A, Kirchner T (2009) The treatment of colorectal carcinoma with monoclonal antibodies: the importance of KRAS mutation analysis and EGFR status. Deutsches Arzteblatt Int 106(12):202-206

Van Cutsem E, Kohne CH, Lang I, Folprecht G, Nowacki MP, Cascinu $S$ et al (2011) Cetuximab plus irinotecan, fluorouracil, and leucovorin as first-line treatment for metastatic colorectal cancer: updated analysis of overall survival according to tumor KRAS and BRAF mutation status. J Clin Oncol 29(15):2011-2019

Yamauchi M, Morikawa T, Kuchiba A, Imamura Y, Qian ZR, Nishihara $R$ et al (2012a) Assessment of colorectal cancer molecular features along bowel subsites challenges the conception of distinct dichotomy of proximal versus distal colorectum. Gut 61(6):847-854

Yamauchi M, Lochhead P, Morikawa T, Huttenhower C, Chan AT, Giovannucci E et al (2012b) Colorectal cancer: a tale of two sides or a continuum? Gut 61(6):794-797 